\title{
Image Fusion of Multislice Spiral CT with Magnetic Resonance Imaging (MRI) in the Diagnosis and Nursing of Malignant Bone Diseases Using ANOVA
}

\author{
Meimei Luo $\mathbb{D}^{1},{ }^{1}$ Run Yang $\mathbb{D},{ }^{2}$ Haijie Zhang $\mathbb{D}^{3},{ }^{3}$ Yi Wang $\mathbb{D}^{4},{ }^{4}$ and Shengmei Gao $\mathbb{D}^{5}$ \\ ${ }^{1}$ Department of Orthopedics, Xijing Hospital, Air Force Medical University, Xi'an 710032, Shaanxi Province, China \\ ${ }^{2}$ Department of Preventive Health Care, Yantai City Qishan Hospital, Shandong, Yantai 264001, China \\ ${ }^{3}$ Department of Orthopaedic, Guangrao County People's Hospital, Shandong 257300, China \\ ${ }^{4}$ Department of Medical Imaging, 74 Group Military Hospital of People's Liberation Army, Guangzhou 510318, China \\ ${ }^{5}$ Operation Room, Linyi Cancer Hospital, Linyi, Shandong 276000, China \\ Correspondence should be addressed to Shengmei Gao; dominicman@mail.sdu.edu.cn
}

Received 16 June 2021; Revised 11 July 2021; Accepted 20 July 2021; Published 29 July 2021

Academic Editor: Gustavo Ramirez

Copyright (c) 2021 Meimei Luo et al. This is an open access article distributed under the Creative Commons Attribution License, which permits unrestricted use, distribution, and reproduction in any medium, provided the original work is properly cited.

\begin{abstract}
This work aimed to analyze the diagnostic value of dynamic scanning of multislice spiral computed tomography (MSCT) and magnetic resonance imaging (MRI) for benign and malignant bone tumor and nursing intervention. 108 patients with bone tumor were selected as the research objects, all of which underwent MSCT and MRI scans. The accuracy, sensitivity, and specificity of MSCT, MRI, and MSCT + MRI for identifying benign and malignant bone tumors and nursing care were calculated, as well as the diagnostic accuracy of MSCT, MRI, and MSCT + MRI for different bone tumor pathological types. The results showed that the accuracy of MSCT + MRI (97.56\%) in distinguishing benign and malignant bone lesions was remarkably higher relative to that of MSCT $(85.91 \%)$ and MRI $(89.85 \%)(P<0.05)$. The sensitivity and specificity of MSCT + MRI $(94.85 \% ; 90.52 \%)$ in distinguishing benign and malignant bone lesions were obviously greater in contrast to those of MSCT (83.66\%; 79.05\%) and MRI (86.02\%; 81.17\%) $(P<0.05)$. The malignant misdiagnosis rate and malignant missing report rate of MSCT + MRI in distinguishing benign and malignant bone lesions were inferior to those of MSCT and MRI notably $(P<0.05)$. The accuracy of MSCT + MRI in distinguishing osteosarcoma, giant-cell tumor of bone (GCT), bone cyst, and osteofibrous dysplasia (OFD) was evidently higher versus that of MSCT and MRI $(P<0.05)$. The accuracy of MSCT + MRI in distinguishing osteofibroma and ganglioneuroma was greatly higher than that of MSCT and MRI $(P<0.05)$. The accuracy of MSCT + MRI in distinguishing osteofibroma and ganglioneuroma was $68.64 \%$ and $71.63 \%$, respectively. In short, in contrast to the single MSCT and MRI examination, MSCT combined with MRI detection can effectively improve the accuracy of judgment for benign and malignant bone tumor lesions and nursing care and had higher sensitivity and specificity. MSCT combined with MRI had better performance in identifying osteosarcoma, GCT, bone cyst, and OFD but poor performance in osteofibroma and ganglioneuroma.
\end{abstract}

\section{Introduction}

Bone lesions mainly refer to bone structure or sclerotin lesions. With the rapid development of the national economy, there are more and more bone diseases caused by different factors, for example, years of strain, trauma, or bone degeneration and endocrine disorders with age, leading to bone lesions [1-3]. Moreover, clinically, due to the different lesions of the patient's bones, the clinical manifestations are also different. If a fracture occurs after being subjected to direct external force, needle-like pain at the injured site will not be felt. Moreover, there will be obvious local swelling, positive tenderness, positive percussion pain, and so on, and the joints adjacent to the fracture will also have dysfunction [4]. Bone lesions generally include cervical spondylosis, lumbar disc herniation, bone hyperplasia, osteoporosis, osteoarthritis, fractures, and bone tumor. Among which, bone tumor is a common tumor 
disease that occurs in the bone or its accessory tissues. The bone lesions can be divided into benign lesions and malignant lesions according to the degree of invasion. Different disease severity accompanied by different targeted treatment methods will also affect the quality of patient prognosis [5, 6]. Malignant skeletal lesions may cause severe pain mechanisms due to the development of the disease and various adverse reactions after treatment, which can trigger negative emotions such as depression, pessimism, and despair. Therefore, it is very important for patients to receive psychological and pain care. Therefore, it is very important to diagnose benign and malignant bone diseases at an early stage.

In the diagnosis of clinical bone lesions, X-ray is a relatively traditional examination method, which has the advantages of low cost and easy operation. Unfortunately, because of the low display resolution, projection position, overlapping parts, and so on, sometimes there will be a higher missed diagnosis rate $[7,8]$. In recent years, with the rapid development of microelectronics and computer technology, multislice spiral computed tomography (MSCT) is gradually utilized in clinical examinations. It can obtain tomographic images by reconstructing the sagittal plane, coronal plane, and any cut plane, to clearly show the internal and marginal situation of the organization and provide corresponding reference information for subsequent clinical diagnosis. Moreover, due to the fast scanning speed, it can perform quantitative scanning diagnosis of heart beat, coronary artery, and its branch calcification, which is a noninvasive examination method $[9,10]$. Magnetic resonance imaging (MRI) is a technique that adopts magnetic fields and radio wave energy pulses to image and inspect human internal organs and structures. In many cases, MRI can provide more information than X-ray, ultrasound, or CT and has the advantages of multiple parameters, high resolution, and accurate display $[11,12]$. With the application of high-performance coils and the optimization of scanning sequences in recent years, MRI has been widely adopted in the diagnosis of bone lesions. At present, most of the research studies on MSCT and MRI are limited to a single index, and there are few studies on the combined application of the two [13]. Therefore, this work intended to utilize MSCT combined with MRI to explore the diagnosis and treatment of malignant bone lesions in bone tumor.

In summary, the clinical adoption of MSCT and MRI is of great value. Based on which, 108 patients with bone tumor were taken as objects, who underwent MSCT and MRI scans. By comparing the accuracy, sensitivity, and specificity of MSCT, MRI, and MSCT + MRI in the identification of benign and malignant bone lesions and identifying the accuracy of different bone tumor pathological types, the application value of enhanced CT combined with MRI was comprehensively evaluated in the diagnosis and nursing of malignant bone diseases.

\section{Materials and Methods}

2.1. Selection of Research Objects. 108 bone tumor patients who were admitted to the hospital from January 15, 2019, to January 10, 2020, were selected as the research objects, and their age range was 20-71 years. Both MSCT and MRI scans were performed. The study had been approved by the Medical Ethics Committee of Hospital, and the patients and their families understood the study and signed an informed consent form.

Inclusion criteria include the following: (1) patients who had not received relevant treatment before; (2) patients without pathological fractures; (3) patients who had not undergone needle biopsy in the past month; (4) patients with complete clinical data; and (6) patients without contraindications to CT examination.

Exclusion criteria include the following: (1) patients older than 71 years; (2) patients who had been treated with antitumor therapy; (3) patients with contraindications to MRI scanning; (4) patients with mental illness; (5) patients gave up halfway during examination; and (6) patients with poor compliance with examination.

2.2. Imaging Examination Methods. In this study, a 64-slice spiral CT scanner manufactured by General Electric was adopted to perform enhanced CT scan on patients with the contrast enhancer of $300 \mathrm{mg} / \mathrm{mL}$ Omnipaque. Image collection was carried out at the central position of the tumor. Scan parameters are as follows: tube voltage was $120 \mathrm{kV}$, tube current was $240 \mathrm{~mA}$, pitch was 0.85 , thickness was $0.65 \mathrm{~mm}$, matrix of $521 \times 521$, scanning speed was $1 \mathrm{~s} /$ week, and scan time of $100 \mathrm{~s} .1 .5 \mathrm{~mL} / \mathrm{kg}$ of nonionic iodine contrast agent was injected into the forearm with an automatic highpressure CT syringe.

Open Mark 5000 permanent magnetic resonance imager produced by Shenzhen Anke High Technology Co., Ltd. was utilized to perform MRI scanning on the patients. The joint and the limb may adopt the body part array coil or the body part wrapping flexible coil and the body part winding coil. Patient was put in supine position with the foot advanced. The examination part was put in the center of the coil, and coronal plane and cross-sectional scan of the hip and shoulder joints were taken. The scanning parameters were as follows: the layer thickness of $5 \mathrm{~mm}$, spacing of $0.5 \mathrm{~mm}$, pulse train repetition time of $750 \mathrm{~ms}$, and echo time of $10 \mathrm{~ms}$.

2.3. Image Analysis. Two senior radiologists independently reviewed MSCT images and MRI images to determine the disease degree of bone tumor lesions. MSCT image benign and malignant diagnostic criteria were as follows. The image showed signs of bone destruction such as osteolytic or osteogenic changes, and the image had sharp edges, and it showed whether there were characteristic manifestations such as calcification and ossification in the lesion. Diagnostic criteria for benign and malignant MRI images were as follows. The benign bone tumors showed expansive bone destruction, and T1WI (T1-weighted imaging) was mostly low signal. The T2WI (T2-weighted imaging) usually showed medium and high signal, and the boundary between the tumor bone and the normal bone was sharp, without periosteal reaction, and the surrounding masses were clear. Malignant bone tumors usually showed signals ranging from medium to high on T1WI and T2WI according to the 
different tissue components in the tumor. Malignant bone tumors usually showed invasive destruction of bone destruction and blur the boundary with normal bone tissue. The bone destruction area usually showed low signal on T1WI. Most of the cortical bone showed medium signal, and the T2WI showed high signal.

2.4. Observation Indexes. The general information of the selected patients' (age, body mass index (BMI), course of disease, and male to female ratio), MSCT, and MRI imaging data were recorded. The accuracy, sensitivity, specificity, malignant misdiagnosis rate, and malignant missing report rate of MSCT, MRI, and MSCT + MRI for distinguishing benign and malignant bone tumors and nursing care were also recorded. The diagnostic accuracy of MSCT, MRI, and MSCT + MRI for different bone tumor pathological types was calculated.

2.5. Statistical Methods. The data in this study were analyzed by SPSS19.0 version statistical software, the measurement data were expressed as mean \pm standard deviation $(\bar{x} \pm s)$, and the count data as percentage (\%). The age, BMI, course of disease, and male-female ratio of the selected patients were compared by analysis of variance. Comparison of accuracy, sensitivity, specificity, malignant misdiagnosis rate, and malignant missing report rate of MSCT, MRI, and MSCT + MRI for distinguishing benign and malignant bone tumors and nursing care was conducted via pair $t$ test, which was also adopted to compare the diagnosis accuracy of different bone tumor pathological types. The difference was statistically significant at $P<0.05$.

\section{Results}

3.1. Descriptive Statistics of Basic Data of Selected Patients. Table 1 shows the descriptive statistics of the basic data of selected patients. In terms of gender, the proportion of male patients $(64.54 \%)$ was evidently greater than that of female patients (35.46\%). In terms of age, patients younger than 30 years old accounted for the most (41.75\%), followed by patients $30-50$ years old (28.83\%). In terms of BMI, patients between 18.5 and $22.9 \mathrm{~m}^{2} / \mathrm{kg}$ accounted for the most (46.72\%), followed by patients less than $18.5 \mathrm{~m}^{2} / \mathrm{kg}(36.45 \%)$ and patients greater than $22.9 \mathrm{~m}^{2} / \mathrm{kg}(16.83 \%)$. In terms of disease course, the proportion of patients from 4 to 10 months was the largest $(46.07 \%)$, followed by patients with course less than 4 months (35.26\%) and more than 10 months (18.67\%).

Figures 1 and 2 show MSCT and MRI images of a male patient with bone tumor (aged 34 years old). On CT images, the tumor was manifested as peripheral sclerosis with a clear center, most of which were in the cortex. Tumor nests were with calcification, flocculent density increased, and no periosteal reaction was seen on the outer edge of the cortical bone. The MRI image showed that the tumor nest had a very rich blood supply. When the lesion was located in the bone marrow or adjacent to the joint, there was no sclerosis edge.
TABLE 1: Basic situation of selected patients.

\begin{tabular}{|c|c|c|c|c|}
\hline Variable & Classification & $\begin{array}{c}\text { Percentage } \\
(\%)\end{array}$ & $x^{2}$ & $P$ \\
\hline Gender & $\begin{array}{c}\text { Male } \\
\text { Female }\end{array}$ & $\begin{array}{l}64.54 \\
35.46\end{array}$ & 4.115 & 0.029 \\
\hline Age (year) & $\begin{array}{c}<30 \\
30-50 \\
50-65 \\
>65\end{array}$ & $\begin{array}{c}41.75 \\
28.83 \\
19.86 \\
9.56\end{array}$ & 5.873 & 0.013 \\
\hline BMI $\left(\mathrm{m}^{2} / \mathrm{kg}\right)$ & $\begin{array}{c}<18.5 \\
18.5-22.9 \\
>22.9\end{array}$ & $\begin{array}{l}36.45 \\
46.72 \\
16.83 \\
\end{array}$ & 7.328 & 0.007 \\
\hline $\begin{array}{l}\text { Course of disease } \\
\text { (month) }\end{array}$ & $\begin{array}{c}<4 \\
4-10 \\
>10\end{array}$ & $\begin{array}{l}35.26 \\
46.07 \\
18.67\end{array}$ & 3.285 & 0.021 \\
\hline
\end{tabular}

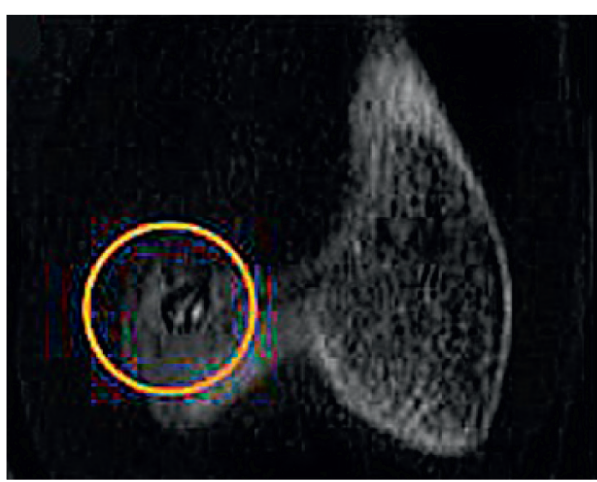

FIGURE 1: MSCT image of a male patient with malignant bone tumor (aged 34 years).

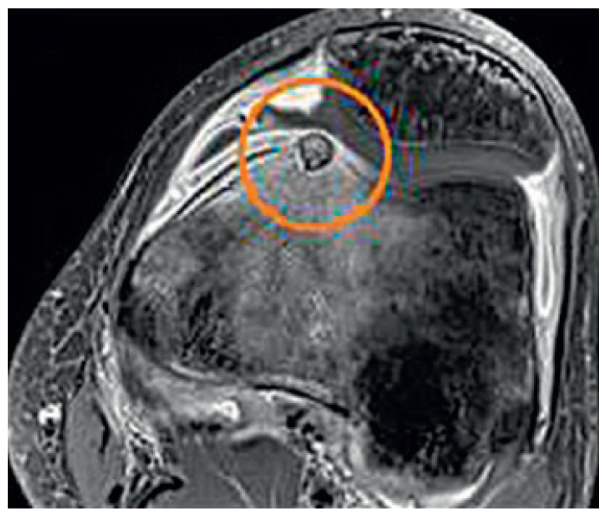

FIGURE 2: MRI image of a male patient with malignant bone tumor (aged 34 years).

3.2. Contrast of Accuracy, Sensitivity, and Specificity of Different Detection Methods in Distinguishing Benign and Malignant Bone Lesions. Figure 3 shows the accuracy comparison of MSCT combined with MRI in identifying benign and malignant bone lesions. The accuracy of MSCT to distinguish benign and malignant skeletal lesions was $85.91 \%$, that of MRI was $89.85 \%$, and that of MSCT + MRI was $97.56 \%$. Among which, the accuracy of MSCT + MRI in distinguishing benign and malignant bone lesions was 


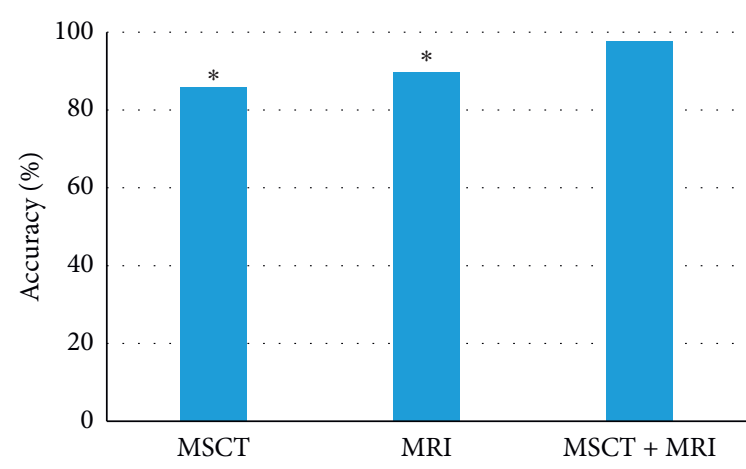

FIGURE 3: Contrast of accuracy of MSCT combined with MRI in distinguishing benign and malignant bone lesions. Note. ${ }^{*}$ indicates considerable difference relative to MSCT + MRI $(P<0.05)$.

greatly higher in contrast to that of MSCT and MRI, and the difference was very notable $(P<0.05)$.

Figure 4 presents the sensitivity and specificity comparison of MSCT combined with MRI in the identification of benign and malignant bone lesions. The sensitivity of MSCT and MRI to distinguish benign and malignant bone lesions was $83.66 \%$ and $86.02 \%$, respectively, and the specificity was $79.05 \%$ and $81.17 \%$, respectively. The sensitivity of MSCT + MRI to distinguish benign and malignant bone lesions was $94.85 \%$, and the specificity was $90.52 \%$. Among which, the sensitivity and specificity of MSCT + MRI in distinguishing benign and malignant bone lesions were notably better relative to MSCT and MRI, with considerable difference $(P<0.05)$.

\subsection{Contrast of Malignant Misdiagnosis Rate and Malignant} Missing Report Rate of Different Detection Methods. Figure 5 shows the comparison of the malignant misdiagnosis rate and the malignant missing report rate of MSCT combined with MRI to identify benign and malignant bone lesions. The malignant misdiagnosis rate of MSCT for identifying benign and malignant bone lesions was $6.08 \%$, and the malignant missing report rate was $8.44 \%$. The malignant misdiagnosis rate and the malignant missing report rate of MRI were $5.22 \%$ and $6.96 \%$, respectively, and those of MSCT + MRI were $3.87 \%$ and $4.31 \%$, respectively. Among which, the malignant misdiagnosis rate and the malignant missing report rate of MSCT + MRI in distinguishing benign and malignant bone lesions were remarkably inferior to MSCT and MRI, and the difference was evident $(P<0.05)$.

\subsection{Contrast of the Accuracy of Different Detection Methods to} Identify the Pathological Type of Bone Tumor. Figure 6 shows the accuracy comparison of MSCT combined with MRI in distinguishing osteosarcoma and GCT. The accuracy of MSCT identifying osteosarcoma was $83.86 \%$, and the accuracy of identifying GCT was $84.2 \%$. The accuracy of MRI identifying osteosarcoma was $85.47 \%$, and the accuracy of identifying GCT was $85.11 \%$. MSCT + MRI showed $93.28 \%$ accuracy in identifying the osteosarcoma and $94.06 \%$

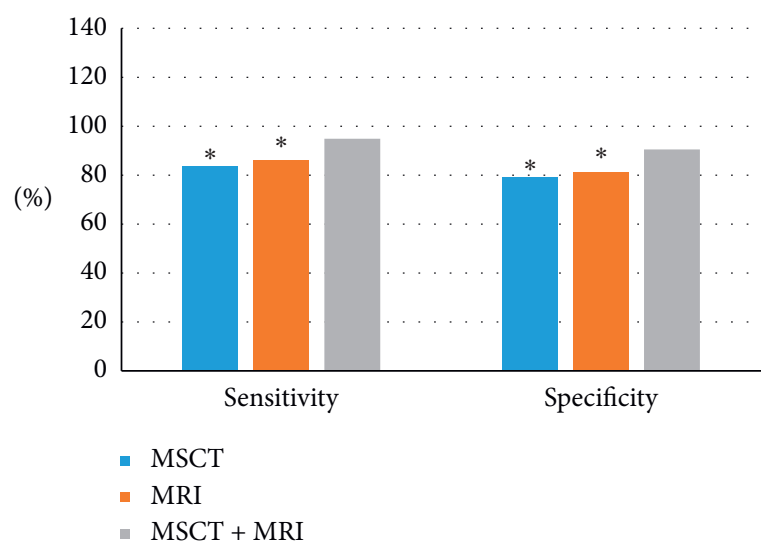

FIGURE 4: Contrast of sensitivity and specificity of MSCT combined with MRI in distinguishing benign and malignant bone lesions. Note. ${ }^{*}$ indicates considerable difference relative to MSCT + MRI $(P<0.05)$.

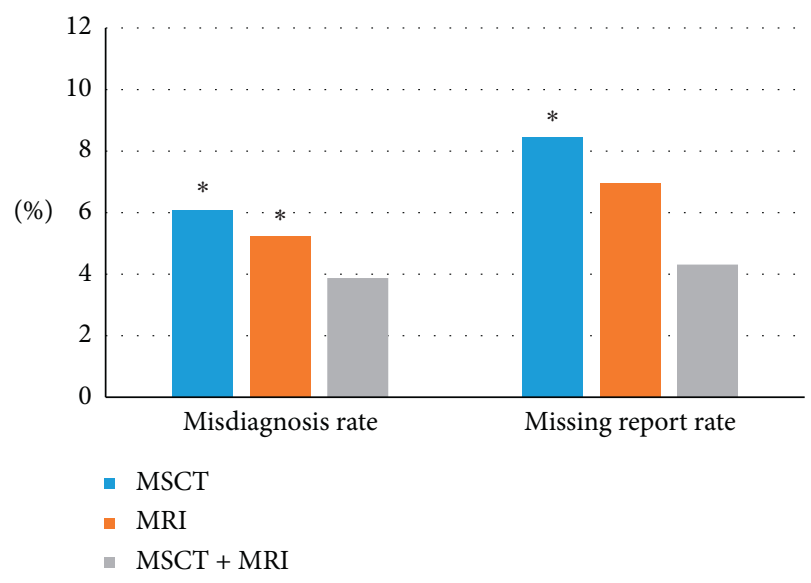

FIgURE 5: Contrast of malignant misdiagnosis rate and malignant missing report rate in the identification of benign and malignant bone lesions by MSCT combined with MRI. Note. ${ }^{*}$ indicates considerable difference relative to MSCT + MRI $(P<0.05)$.

(\%)

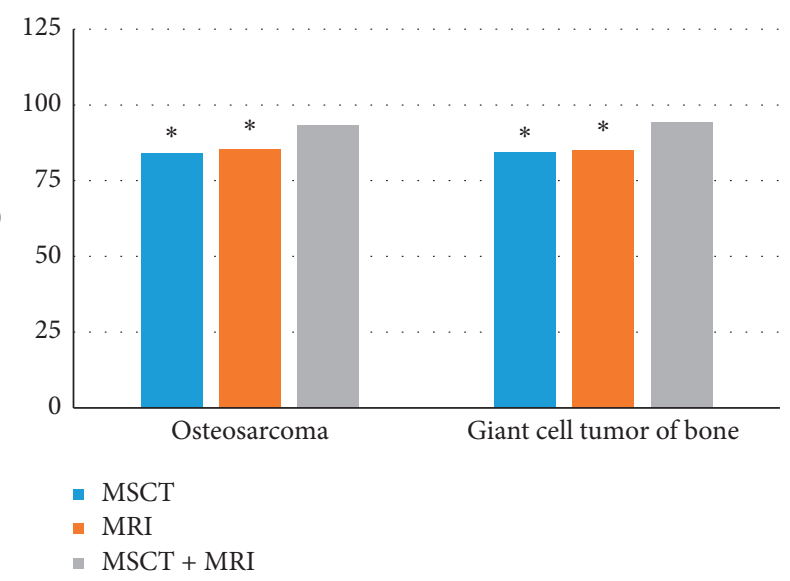

Figure 6: Contrast of accuracy of different detection methods for identifying osteosarcoma and GCT. Note. ${ }^{*}$ indicates considerable difference relative to MSCT + MRI $(P<0.05)$. 
accuracy in identifying GCT. Among which, the accuracy of MSCT + MRI in distinguishing osteosarcoma and GCT was highly greater in contrast to that of MSCT and MRI, with obvious difference $(P<0.05)$.

Figure 7 shows the accuracy comparison of MSCT combined with MRI in identifying bone cyst and OFD. The accuracy of MSCT identifying bone cyst was $82.17 \%$, and the accuracy of identifying OFD was $82.08 \%$. The accuracy of MRI identifying bone cyst was $80.95 \%$, and the accuracy of identifying OFD was $84.14 \%$. The accuracy of MSCT + MRI in identifying bone cyst was $89.33 \%$, and the accuracy of identifying OFD was $88.55 \%$. Among which, the accuracy of MSCT + MRI in distinguishing bone cyst and OFD was obviously higher versus that of MSCT and MRI $(P<0.05)$.

Figure 8 compares the accuracy of MSCT combined with MRI in identifying the osteofibroma and ganglioneuroma. The accuracy of MSCT in identifying osteofibroma and ganglioneuroma was $58.55 \%$ and $61.51 \%$, respectively. The accuracy of MRI in identifying osteofibroma and ganglioneuroma was $54.91 \%$ and $59.74 \%$, respectively. MSCT + MRI showed $68.64 \%$ accuracy in identifying the osteofibroma and $71.63 \%$ accuracy in identifying ganglioneuroma. Among which, the accuracy of MSCT + MRI in identifying osteofibroma and ganglioneuroma was evidently better relative to MSCT and MRI, and the difference was considerable $(P<0.05)$.

\section{Discussion}

Bone tumor generally includes basic bone tissue tumors and bone accessory tissue tumors, which are relatively common tumor diseases. In malignant bone tumors, osteosarcoma generally has the highest incidence, accounting for about $42 \%$, followed by GCT, which is about $13 \%[14,15]$. How to diagnose malignant bone tumor with appropriate examination methods in clinic is also a hot topic at present. Therefore, 108 patients with bone tumor were selected as the research objects, and they all underwent MSCT and MRI scans. Moreover, the accuracy, sensitivity, and specificity of MSCT, MRI, and MSCT + MRI were compared regarding identifying benign and malignant bone lesions and nursing care. The results showed that the accuracy of MSCT + MRI in distinguishing benign and malignant bone lesions was obviously higher versus that of MSCT and MRI, and the difference was evident $(P<0.05)$. It was similar to the results of Caers et al. [16], showing that compared with the single MSCR and MRI, MSCT combined with MRI detection can more effectively improve the accuracy of the diagnosis of malignant bone tumor. The sensitivity and specificity of MSCT + MRI in distinguishing benign and malignant bone lesions were also better than those of MSCT and MRI $(P<0.05)$, revealing that MSCT combined with MRI detection had high sensitivity and specificity for the diagnosis of malignant bone tumor and was of adoption value. The malignant misdiagnosis rate and malignant missing report rate of MSCT + MRI in distinguishing benign and malignant bone lesions were notably inferior to those of MSCT and MRI, with remarkable difference $(P<0.05)$. It was consistent with the results of Carlbom et al. [17], indicating that MSCT combined with MRI detection can better improve the misdiagnosis and

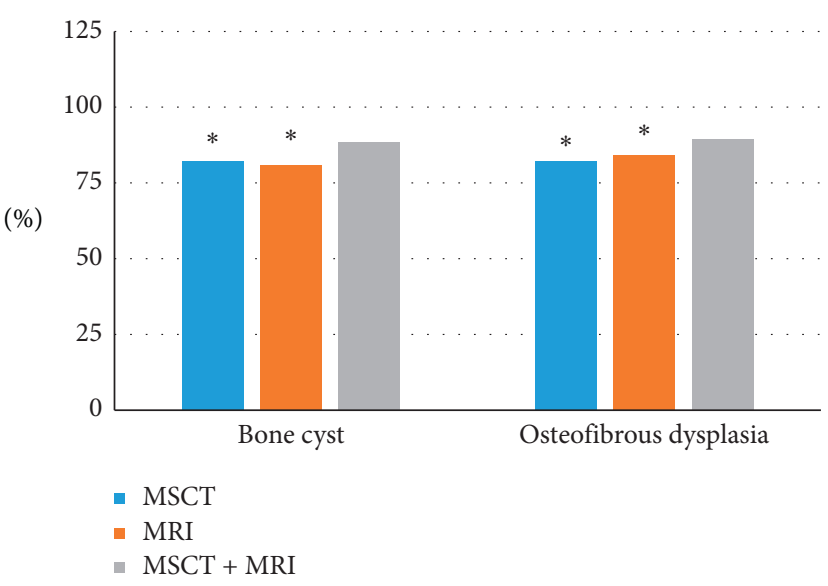

Figure 7: Contrast of accuracy of different detection methods to identify bone cyst and OFD. Note. ${ }^{*}$ indicates considerable difference relative to MSCT + MRI $(P<0.05)$.

(\%)

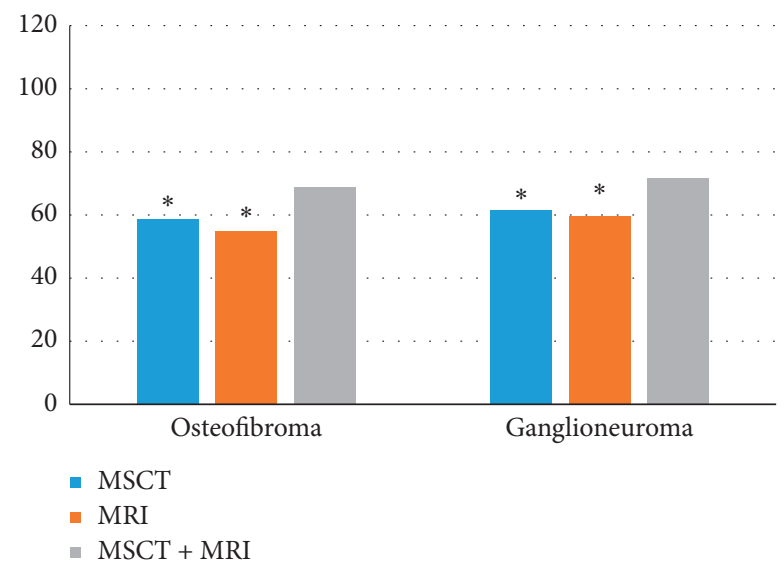

FIgure 8: Contrast of accuracy of different detection methods for identifying osteofibroma and ganglioneuroma. Note. ${ }^{*}$ indicates considerable difference relative to MSCT + MRI $(P<0.05)$.

missed diagnosis of single MSCT and MRI examination of malignant bone tumor.

After the accuracy of MSCT combined with MRI for different pathological types of bone tumor was analyzed, it was found that the accuracy of MSCT + MRI in distinguishing osteosarcoma and GCT was greatly higher in contrast to that of MSCT and MRI $(P<0.05)$, which was similar to the results of Leynes et al. [18], indicating that MSCT combined with MRI had a better diagnostic effect for osteosarcoma and GCT, and the accuracy had been greatly improved [19]. The accuracy of MSCT + MRI in distinguishing bone cyst and OFD was also the optimal one among all methods $(P<0.05)$, which also suggested that MSCT combined with MRI was better than single MSCT and MRI detection for the diagnosis of bone cyst and OFD. In addition, the accuracy of MSCT + MRI in distinguishing osteofibroma and ganglioneuroma was also higher versus that of MSCT and MRI $(P<0.05)$. However, the accuracy of MSCT + MRI in distinguishing osteofibroma and ganglioneuroma was $68.64 \%$ and $71.63 \%$, respectively. The results 
were different from those of Ellmann et al. [20], and the reason may be that the sample size of patients was quite different, and the adopted contrast agent for CT scan was also different. Therefore, although MSCT + MRI can improve the accuracy of single MSCT and MRI detection for osteofibroma and ganglioneuroma, the diagnostic performance of these two malignant tumors was poor and the accuracy was relatively low.

\section{Conclusion}

108 cases of bone tumor patients were selected as the research objects, and all of them underwent MSCT and MRI scans. Then, the accuracy, sensitivity, and specificity of MSCT, MRI, and MSCT + MRI were compared in terms of identifying benign and malignant bone lesions and nursing care, and the diagnosis accuracy of different bone tumor pathological types was compared, too. It was disclosed that compared with single MSCT and MRI detection, MSCT combined with MRI can effectively improve the accuracy of identifying benign and malignant bone tumor lesions and had higher sensitivity and specificity. MSCT combined with MRI had better performance in identifying osteosarcoma, GCT, bone cyst, and OFD but poor performance in osteofibroma and ganglioneuroma. However, the sample size of the patients selected is small, resulting in fewer bone tumor cases such as soft osteosarcoma, bone schwannoma, and ganglioneuroma. Later, the patient sample size will be considered to expand to further explore the clinical effects of MSCT combined with MRI. In conclusion, the results of this article provide good experimental support for the combined diagnosis of clinical imaging of bone tumors and malignant bone lesions.

\section{Data Availability}

No data were used to support this study.

\section{Conflicts of Interest}

The authors declare that there are no conflicts of interest.

\section{Acknowledgments}

This work was supported by the Key Funding Projects for Independent Innovation of Health System Research in Putuo District, Shanghai (SH201741).

\section{References}

[1] J. Zhou, Z. Gou, R. Wu, Y. Yuan, G. Yu, and Y. Zhao, "Comparison of PSMA-PET/CT, choline-PET/CT, NaF-PET/ $\mathrm{CT}$, MRI, and bone scintigraphy in the diagnosis of bone metastases in patients with prostate cancer: a systematic review and meta-analysis," Skeletal Radiology, vol. 48, no. 12, pp. 1915-1924, 2019 Dec.

[2] L. A. Castroneves, G. Coura Filho, R. M. C. de Freitas et al., "Comparison of 68Ga PET/CT to other imaging studies in medullary thyroid cancer: superiority in detecting bone metastases," Journal of Clinical Endocrinology \& Metabolism, vol. 103, no. 9, pp. 3250-3259, 2018 Sep.
[3] G. K. Azad, B. P. Taylor, A. Green et al., "Prediction of therapy response in bone-predominant metastatic breast cancer: comparison of [18F] fluorodeoxyglucose and [18F]-fluoride PET/CT with whole-body MRI with diffusion-weighted imaging," European Journal of Nuclear Medicine and Molecular Imaging, vol. 46, no. 4, pp. 821-830, 2019 Apr.

[4] H. D. Zacho, J. B. Nielsen, A. Afshar-Oromieh et al., "Prospective comparison of $68 \mathrm{Ga}$-PSMA PET/CT, 18F-sodium fluoride PET/CT and diffusion weighted-MRI at for the detection of bone metastases in biochemically recurrent prostate cancer," European Journal of Nuclear Medicine and Molecular Imaging, vol. 45, no. 11, pp. 1884-1897, 2018 Oct.

[5] H. Y. Choi, D. Y. Yoon, E. S. Kim et al., "Diagnostic performance of CT, MRI, and their combined use for the assessment of the direct cranial or intracranial extension of malignant head and neck tumors," Acta Radiologica, vol. 60, no. 3, pp. 301-307, 2019 Mar.

[6] J. Löfgren, J. Mortensen, S. H. Rasmussen et al., "A prospective study comparing 99mTc-hydroxyethylenediphosphonate planar bone scintigraphy and whole-body SPECT/CT with 18F-fluoride PET/CT and 18F-fluoride PET/ MRI for diagnosing bone metastases," Journal of Nuclear Medicine, vol. 58, no. 11, pp. 1778-1785, 2017 Nov.

[7] P. Moreau, M. Attal, D. Caillot et al., "Prospective evaluation of magnetic resonance imaging and $[18 \mathrm{~F}]$ fluorodeoxyglucose positron emission tomography-computed tomography at diagnosis and before maintenance therapy in symptomatic patients with multiple myeloma included in the IFM/DFCI 2009 trial: results of the IMAJEM study," Journal of Clinical Oncology, vol. 35, no. 25, pp. 2911-2918, 2017 Sep.

[8] H. Ishiguchi, S. Ito, K. Kato et al., "Diagnostic performance of 18F-FDG PET/CT and whole-body diffusion-weighted imaging with background body suppression (DWIBS) in detection of lymph node and bone metastases from pediatric neuroblastoma," Annals of Nuclear Medicine, vol. 32, no. 5, pp. 348-362, 2018 Jun.

[9] Y. Le, Y. Chen, F. Zhou, G. Liu, Z. Huang, and Y. Chen, "Comparative diagnostic value of $18 \mathrm{~F}$-fluoride PET-CT versus MRI for skull-base bone invasion in nasopharyngeal carcinoma," Nuclear Medicine Communications, vol. 37, no. 10, pp. 1062-1068, 2016 Oct.

[10] T. Wilkman, S. Apajalahti, E. Wilkman, J. Törnwall, and P. Lassus, "A comparison of bone resorption over time: an analysis of the free scapular, iliac crest, and fibular microvascular flaps in mandibular reconstruction," Journal of Oral and Maxillofacial Surgery, vol. 75, no. 3, pp. 616-621, 2017 Mar.

[11] T. R. F. Van Steenbergen, M. Smits, T. W. J. Scheenen et al., "68Ga-PSMA-PET/CT and diffusion MRI targeting for conebeam CT-guided bone biopsies of castration-resistant prostate cancer patients," CardioVascular and Interventional Radiology, vol. 43, no. 1, pp. 147-154, 2020 Jan.

[12] T. Geith, E. Brun, A. Mittone et al., "Quantitative assessment of degenerative cartilage and subchondral bony lesions in a preserved cadaveric knee: propagation-based phase-contrast CT versus conventional MRI and CT," American Journal of Roentgenology, vol. 210, no. 6, pp. 1317-1322, 2018 Jun.

[13] A.-S. Björkman, S. K. Koskinen, M. Lindblom, and A. Persson, "Diagnostic accuracy of dual-energy CT for detection of bone marrow lesions in the subacutely injured knee with MRI as reference method," Acta Radiologica, vol. 61, no. 6, pp. 749-759, 2020 Jun.

[14] C. W. Ding, Q. S. Wang, Q. Y. Guo, J. Zhang, and Z. H. Wang, "CT and MRI features in adult patients with orbital 
subperiosteal abscess secondary to paranasal sinus mucocele," Ear, Nose, \& Throat Journal, vol. 96, no. 7, pp. E1-E6, 2017 Jul.

[15] F. M. Prins, J. M. van der Velden, A. S. Gerlich et al., "Superior target delineation for stereotactic body radiotherapy of bone metastases from renal cell carcinoma on MRI compared to CT," Annals of Palliative Medicine, vol. 6, no. S2, pp. S147-S154, 2017 Dec.

[16] J. Caers, B. Paiva, E. Zamagni et al., "Diagnosis, treatment, and response assessment in solitary plasmacytoma: updated recommendations from a European Expert Panel," Journal of Hematology \& Oncology, vol. 11, no. 1, p. 10, 2018 Jan.

[17] L. Carlbom, J. Caballero-Corbalán, D. Granberg, J. Sörensen, B. Eriksson, and H. Ahlström, "Whole-body MRI including diffusion-weighted MRI compared with 5-HTP PET/CT in the detection of neuroendocrine tumors," Upsala Journal of Medical Sciences, vol. 122, no. 1, pp. 43-50, 2017 Mar.

[18] A. P. Leynes, J. Yang, F. Wiesinger et al., "Zero-echo-time and dixon deep pseudo-CT (ZeDD CT): direct generation of pseudo-CT images for pelvic PET/MRI attenuation correction using deep convolutional neural networks with multiparametric MRI," Journal of Nuclear Medicine, vol. 59, no. 5, pp. 852-858, 2018 May.

[19] I. Jambor, A. Kuisma, S. Ramadan et al., "Prospective evaluation of planar bone scintigraphy, SPECT, SPECT/CT, 18F$\mathrm{NaF}$ PET/CT and whole body 1.5T MRI, including DWI, for the detection of bone metastases in high risk breast and prostate cancer patients: SKELETA clinical trial," Acta Oncologica, vol. 55, no. 1, pp. 59-67, 2016.

[20] S. Ellmann, L. Seyler, J. Evers et al., "Prediction of early metastatic disease in experimental breast cancer bone metastasis by combining PET/CT and MRI parameters to a Model-Averaged Neural Network," Bone, vol. 120, pp. 254261, 2019 Mar. 